\title{
Diagnóstico para el diseño de un programa de intervención psicológica para familias en procesos psico-jurídicos de alto conflicto
}

\section{Diagnosis for the design for a psychological intervention program for families in high conflict psycho-legal proceedings}

Anayancy Silveira Tus

Reyna Faride Peña Castillo

Silvia María Álvarez Cuevas

Universidad Autónoma de Yucatán (UADY)

\section{Resumen}

El presente trabajo expone el diagnóstico realizado sobre actores del sistema de justicia y profesionales del área psico-jurídica familiar para la detección de necesidades de intervención de las familias en procesos jurídicos familiares de alto conflicto. Asimismo, que tal dictamen derive en atención psicológica por orden judicial. Los resultados del diagnóstico dan a conocer las características principales de las familias que se encuentran en estos procesos. En función de estos elementos se definieron las líneas de intervención, así como el diseño, aplicación y evaluación de un programa cuyo objetivo principal fue brindar una alternativa de intervención psico-jurídica bajo un modelo psicoeducativo y de apoyo social. Tal apoyo se proyecta para facilitar la convivencia parento-filial y favorecer la toma de acuerdos entre los padres, además de favorecer el desarrollo óptimo y el bienestar psicológico de las niñas, niños, madres y padres participantes.

Palabras clave: divorcio, apoyo social, derecho familiar, justicia terapéutica.

Nota del autor

Anayancy Silveira Tus, Facultad de Psicología, Universidad de Yucatán (UADY).

La correspondencia en relación con este artículo debe dirigirse a Anayancy Silveira Tus Galaz, Facultad de Psicología, UADY. Carretera Mérida Tizimin S/N, Cholul C.P. 97305, Mérida, Yucatán, México.

Dirección electrónica: anayancy.silveira@gmail.com 


\begin{abstract}
The present paper exposes a diagnosis from the perspective of different actors of the justice system and professionals of family psycho-juridical area about the intervention needs for families in high conflict family legal processes that are in psychological attention by judicial order. Diagnosis results reveal the main characteristics of the families that are in these processes. Based on those results an intervention program was designed, which objective is to provide an alternative of psycho-legal intervention under a psychoeducational and social support model to facilitate parent-filial coexistence and favor the adoption of agreements between parents, as well as to benefit the optimal development and psychological well-being of girls, boys, mothers and fathers participants.
\end{abstract}

Keywords: divorce, social support, family law, therapeutic justice.

El divorcio, una de las formas o factores determinantes de la desintegración familiar, es visto como un problema relevante cuando se toma en cuenta que impacta directamente en la satisfacción de necesidades físicas, afectivas, sociales, económicas y legales de forma óptima y provoca efectos negativos que dificultan, en gran medida, el pleno desarrollo de los integrantes de esta familia. No obstante, si bien es un hecho que el divorcio y separación conforman un proceso complejo, también es cierto que las implicaciones para cada integrante de una familia son diferentes y muchas veces las consecuencias derivan más de cómo se da el proceso que de la separación en sí misma.

Las afectaciones antes señaladas se ven incrementadas cuando existe un alto nivel de conflicto, que de acuerdo a Gil (2011), son aquellos procesos "en donde las partes involucradas, a pesar de que haya transcurrido un tiempo largo desde la separación, no acaban de resolver el conflicto, sino que, al contrario, éste se incrementa o se prolonga indefinidamente" (pag.7). En este sentido, las separaciones y divorcios de alto conflicto, establecen un factor de riesgo para la aparición y cronificación de comportamientos que condicionan y pueden perjudicar el desarrollo psicológico y emocional, así como la adaptación necesaria a la nueva situación, de todos los miembros de la familia (Bonasa et al., 2010).

Resulta, entonces, relevante tener alternativas para solucionar las diferentes problemáticas que se presentan y coadyuvar a que el divorcio sea una oportunidad de crecimiento, no sólo un hecho con efectos negativos en las personas. Tomando esto en consideración, en el ámbito del derecho familiar, se han comenzado a emitir órdenes judiciales de tratamiento para que los hijos y progenitores que atraviesan un proceso de divorcio asistan a intervención o apoyo psicológico. El tratamiento psicológico acentúa el desarrollo de estrategias que pueden ser esenciales para lograr el ajuste necesario, la solución de las distintas problemáticas y la recuperación de los síntomas y afectaciones provocadas por el impacto del alto nivel de conflicto (Tejedor, 2010). 
En nuestro país aún se observa que las opciones de intervención donde se conjugan el aspecto psicológico y jurídico son limitadas. Por ello, este trabajo busca contribuir en la elaboración de estrategias especializadas desde la psicología jurídica y plantea un programa orientado al abordaje de las problemáticas derivadas de un divorcio conflictivo, con el fin de brindar una alternativa de intervención a aquellas familias que, debido al proceso jurídico contencioso, necesiten orientación psicológica y emocional. De esta manera se permitirá no solo incidir en los procesos judiciales con mayores garantías para la resolución y toma de decisiones, sino también favorecer el desarrollo óptimo y el bienestar de las personas que se ven inmersas en procesos de separación y divorcio de alto conflicto.

Es importante resaltar que este trabajo se fundamenta en el enfoque de la justicia terapéutica, que se refiere al estudio y promoción de la ley como un agente de bienestar personal y comunitario (Wexler \& Winick, 1991, en Frías, 2014). Tal acercamiento interdisciplinario entre la psicología y el derecho, indica que el contenido de la ley y su administración pueden tener un efecto positivo o negativo en las personas, cuando es positivo se denomina terapéutico y en sentido contrario antiterapéutico (Frías, 2014). En México, la justicia terapéutica es un concepto poco difundido, pero necesario en su aplicación, en tal contexto, este trabajo se justifica en la medida que pretende contribuir en la construcción de una justicia más terapéutica para los padres e hijos que están inmersos en procesos dentro del sistema familiar y en la difusión y aplicación de esta perspectiva dentro del sistema de justicia de nuestro país. A partir del ordenamiento judicial de atención psicológica y de las necesidades detectadas en este diagnóstico, se buscó implementar un programa que favoreciera el bienestar psicológico de cada una de las partes involucradas en el proceso judicial.

\section{Marco referencial}

\section{Separación y divorcio}

Al tomar la decisión de una separación y en su enfrentamiento, se plantean diversas crisis para cada una de las partes $y$, en general, para el sistema familiar, dado que se requiere de una serie de ajustes psicológicos, afectivos, relacionales, económicos y sociales -los cuales se pueden catalogar como estresantes para toda la familia-, aunque a cada miembro le pueda afectar de forma diferente (Cantón, Cortés, Justicia, \& Cantón, 2014). Estos ajustes implican una reestructuración familiar, además del núcleo conyugal, de las relaciones entre los hijos con sus padres y con las familias extensas. Tal circunstancia da una nueva definición de los roles familiares; la forma en la cual se produzca esta reestructuración será el predictor fundamental del equilibrio de las nuevas relaciones, sobre todo en el nivel parento-filial. Si la separación es conflictiva, las consecuencias de este cambio se vuelven más perjudiciales (De la Torre, 2006). 


\section{La separación y el divorcio en el contexto psico-jurídico}

Diversos elementos convergen para dar lugar a una separación o divorcio con un alto nivel de conflicto. Sobre este aspecto, Bolaños (1998) explica que, así como existen diferentes formas de llevar a cabo una relación de pareja, se pueden sintetizar estilos conyugales diferentes a la hora de abordar la separación. Según el autor, las pautas en la relación que se dieron durante la vida de pareja se traslapan a una nueva situación y con diferentes niveles de intensidad. Se podría predecir cómo determinadas parejas están más encaminadas hacia procesos legales contenciosos, donde el enfrentamiento en el juzgado sustituye al del hogar, o hacia acuerdos más civilizados, en función del estilo relacional que han ido negociando durante su convivencia.

De acuerdo con lo anterior, el proceso de separación, desde la perspectiva legal, se puede dividir en dos tipos: la disolución por mutuo acuerdo, en la cual los mismos cónyuges establecen las condiciones por las cuales se llevará a cabo la separación y, por otro lado, el proceso de tipo contencioso, en el que al no poder establecerse condiciones en común y acuerdos, estos se dejan a la valoración de un juez para la mejor resolución del conflicto. Esta situación suma nuevas consecuencias al conflicto inicial: un mayor coste económico, emocional e incluso psicológico para todos los integrantes de la familia (Fariña \& Arce, 2006).

El proceso legal de una separación contenciosa puede ayudar a regular algunos aspectos psicosociales, al parecer innegociables, sin embargo, a pesar del componente jurídico propicio para resolverlos, no se sustituye el proceso psicosocial; no se pueden dejar de lado los componentes de tipo emocional, afectivos o sociales que es necesario resolver por otras vías más especializadas. Como menciona Bolaños (2000, p. 8), "el tiempo legal y el tiempo psicosocial son diferentes, los procesos emocionales se inician con anterioridad a los trámites legales y finalizan posteriormente", en este mismo sentido, el autor cree probable que las diferentes tareas adaptativas, requeridas para llevar a cabo una adecuada separación en los procedimientos contenciosos, se vean mezcladas, y se obstaculicen entre sí. De ahí que dichas tareas amplíen su campo de expresión al proceso legal, donde se presentan conflictos de pareja y conflictos de padres que requieren soluciones judiciales y psicosociales diferentes.

Sobre este mismo aspecto, Bolaños (1998) recalca que es esperado un cierto nivel de conflicto, pero que éste puede catalogarse como destructivo "cuando conlleva tensión prolongada, produce hostilidad crónica, reduce drásticamente el nivel de vida, perjudica el bienestar psicológico o destruye las relaciones familiares". Dentro de una separación con un alto nivel de conflicto, es posible que se vislumbren o se desarrollen otro tipo de problemáticas que causan daño psicológico a los hijos, ya que dichas separaciones pueden llegar a enmarcarse en escenarios donde existen situaciones de riesgo, maltrato infantil o violencia familiar. 
De la Torre (2006) indica que las separaciones se pueden calificar como conflictivas no solo cuando el litigio va más allá de la disolución matrimonial, sino también cuando existe una falta de cooperación en las funciones parentales de cuidado, educación y bienestar de los hijos, reflejada en las disputas personales y judiciales.

A su vez, para este mismo autor, se pueden catalogar como separaciones conflictivas aquellas que por sí mismas provocan un malestar personal, angustia y disconformidad; cuando las partes permanecen en una actitud de constante perjuicio contra el o en aquellas separaciones donde el impacto del proceso afecta de manera negativa las relaciones entre padres e hijos o se produce una mala adaptación a la ruptura.

Johnston y Roseby (1997) hacen mención de comportamientos y actitudes que pueden presentar los padres involucrados en situaciones de alto conflicto, por ejemplo, grados elevados de ira y desconfianza; incidentes de abuso verbal; agresión física intermitente; dificultades para comunicarse, para cooperar en el cuidado y para concentrarse en las necesidades de sus hijos; así como dificultades para separar, y protegerlos de su propia angustia emocional, ira o de las disputas constantes entre ellos.

Por otra parte, Bonasa y colaboradores (2010) aúnan a lo anterior conductas como las referentes a la distorsión o manipulación de la imagen del otro progenitor con el propósito de alienar a los hijos, las interferencias para que el otro cumpla el régimen de visitas o la imposibilidad para su realización, las campañas de denuncias falsas y cronificación innecesaria del proceso judicial, así como tomar el papel de víctima frente a los hijos para ganar su lealtad y lograr la descalificación y desprestigio del otro progenitor en presencia de los hijos.

En sí, los miembros de la familia, tanto progenitores como hijos, envueltos en una separación o divorcio contencioso y de alto conflicto, presentarán una serie de afectaciones psicológicas que puede influir de manera adversa en el bienestar y en la futura relación entre ellos. Como ya se ha mencionado, estas consecuencias dependen de manera muy significativa de cuáles sean las condiciones y el entorno en los que la ruptura se produce y no del mero hecho de la separación (Fernández \& Godoy, 2005).

Los infantes que atraviesan este proceso pueden presentar, de forma inmediata, estrés emocional y problemas de conducta ante la confusión y aprensión que les provoca el cambio en las relaciones familiares y en su propia vida (Cantón, Cortés, \& Justicia, 2002; Hetherington, 2003; Kelly, 2003, citados en Cortés, 2010). Durante los meses siguiente al divorcio, la mayoría de los niños puede experimentar problemas de tipo externo (como conducta antisocial, agresiva, desobediencia, falta de autorregulación, baja responsabilidad y logro) y, en menor medida, ansiedad, depresión $\mathrm{y}$ problemas en las relaciones sociales (por ejemplo, dificultades con padres, hermanos, iguales y profesores) (Tejedor, 2012).

Los hijos, asimismo, pueden experimentar sentimientos de miedo al abandono, de culpa, de 
negación y comportamientos de suplantación, donde el hijo ocupa el lugar simbólico del progenitor que se ha ido (Tejedor, 2007). Esto último puede ocasionar una sobrecarga de tareas, para la cual los hijos no están psicológica ni evolutivamente preparados. Los vástagos, entonces, se ven obligados a adoptar una postura activa en la situación donde participan de manera forzada, ante la incapacidad de ambos padres, que se encuentran inmersos en sus conflictos personales (Ackerman, 1995; Aguilar, 2007).

Otras consecuencias recurrentes asociadas a la separación de alto conflicto, pueden ser la alienación parental, cuando el niño manifiesta rechazo hacia el progenitor, de manera inducida; y la ilusión de reconciliación, que se caracteriza por mantener, en contra de toda evidencia, la esperanza de la reconciliación de los padres, lo cual agudiza la situación y no les permite lograr una adaptación y aceptación de la separación, así como ser conscientes de sus implicaciones (Fariña, Seijo, Arce, \& Novo, 2002).

Específicamente, para los padres, el divorcio conflictivo puede generar altos índices de estrés, ansiedad y agotamiento físico o emocional, ya que como menciona Kelly (2003, citado en Muñoz, 2009), la decisión de separarse legalmente llega con frecuencia después de un largo y doloroso proceso. El conflicto, la ansiedad, una pobre comunicación, la tristeza o el enfado padecidos durante dicho proceso pueden convertirse en parte habitual del día a día. Muñoz (2009) refiere, a su vez, que en este periodo se combina la lucha entre las partes, con el proceso legal que acompaña a la ruptura y otros factores, cuyas congunción puede dejar secuelas en ambos y una gran incertidumbre.

Según, De la Torre (2005), en ocasiones el origen del enfrentamiento se sitúa en el instante en el que no se ha resuelto la separación emocional con el excónyuge. En este caso, se pueden vislumbrar sentimientos como el odio, la contrariedad, la venganza, la revancha, el resarcimiento por el dolor sufrido, o simplemente la incomprensión o el desacuerdo por la separación. Tales sentimientos pueden fomentar esas conductas de enfrentamiento y conflicto, con el fin de mantener unida/ desunida esa relación, como si el conflicto fuera, entonces, el referente de la unión. Cuando esta circunstancia todavía persiste, según señala el autor, es más probable que se utilice a los hijos como parte de ese enfrentamiento dual paterno-materno hasta cierto tiempo después de la separación, es decir, mientras cada uno no asimile dicho proceso y no supere dicha vivencia personal.

Además de las consecuencias descritas, también existe una relación entre la exposición a estas situaciones de alto conflicto y diversos tipos de trastornos psicopatológicos y elevaciones significativas del estrés y de ansiedad, tanto en los niños como en los adultos (Grych \& Fincham, 1990, citado en Arch, 2010).

\section{Programas de intervención en el derecho de familia}

Alrededor de los años 70, en Estados Unidos surgieron los programas psicoeducativos para 
ayudar a la familia a superar de manera eficaz la ruptura, además de prevenir comportamientos de los progenitores que impidan a los hijos adaptarse al divorcio (Deutsch, 2008). De forma genérica, tienen por objetivo formar a las parejas con tendencia a separarse, para que se centren en el mejoramiento de su trato conyugal, conduciéndolas a una relación de cooperación parental ajena al conflicto. Con respecto a lo anterior, Kirby (1998), menciona que dentro de los programas de intervención, se han de enseñar los beneficios de la colaboración parental y las responsabilidades del padre custodio $\mathrm{y}$ del no custodio, las reacciones de los hijos al divorcio y las consecuencias del conflicto y de la alienación parental, con el fin de incrementar el bienestar de todos los miembros de la familia. Cabe mencionar que estos programas han sido exitosos en el ámbito anglosajón desde los años ochenta y noventa, hasta la actualidad.

Por su parte, Cantón y Justicia (2007), han recopilado una serie de programas para prevenir los problemas de adaptación de los hijos divorciados; dichas intervenciones han surgido principalmente en Estados Unidos y se han implementado en contextos judiciales. Los autores clasifican estos programas en dos tipos: programas educativos para padres divorciados e intervenciones centradas en el niño. Los programas educativos, generalmente, tienden a la reducción del conflicto destructivo al que se ve expuesto el infante y a fomentar una relación adecuada entre los padres e hijos(as); estas intervenciones se han diseñado en función del grupo al cual estén dirigidos, por ejemplo: progenitores custodios y progenitores no custodios. Referente a las intervenciones centradas en el infante, Cantón y Justicia (2007), señalan que los programas psicoeducativos intentan ayudar a los hijos, al aliviar sentimientos negativos, concepciones erróneas y problemas prácticos relacionados con el divorcio, y resaltar, a su vez, la utilidad del apoyo social para los niños(as) en estos grupos de intervención.

No obstante, se observa que en Latinoamérica existe un amplio desconocimiento sobre dichos programas de intervención, lo cual expresa una escasa implementación, considerando que en algunos países son simplemente inexistentes. En lo que respecta a México, los programas a nivel nacional y local, con enfoque en la intervención psicológica con familias que se encuentren en divorcios y separaciones de alto conflicto, son escasos. Tal situación compromete la creación urgente de este tipo de programas para disminuir los efectos ya antes dichos sobre los integrantes de las familias mexicanas (Arce \& Fariña, 2007).

\section{Método}

El presente trabajo está enmarcado en una metodología cualitativa que, según Hernández, Fernández y Baptista (2010), se enfoca en comprender y profundizar los fenómenos, explorándolos desde la perspectiva de los participantes en un ambiente natural y en relación con el contexto. Por lo general, se selecciona cuando se busca comprender la perspectiva 
de los participantes acerca de los fenómenos que los rodean, así como para profundizar en sus experiencias, perspectivas, opiniones $\mathrm{y}$ significados.

\section{Participantes}

Se realizaron entrevistas a dos juezas familiares, del sistema oral y tradicional, respectivamente, ambas del Primer Departamento Judicial del Estado de Yucatán y a dos psicólogas que intervienen en el área de psicología jurídica familiar, tanto en evaluación psicológica forense como intervención psicológica especializada en el ámbito familiar. Las entrevistas de las juezas y de la psicóloga con funciones de evaluación fueron realizadas en la sede de los juzgados familiares del Estado.
Por otra parte, se entrevistó en un consultorio particular a la psicóloga que desempeña funciones de intervención especializada en el ámbito privado.

\section{Técnicas e instrumentos}

Se utilizó como técnica de recolección de datos la entrevista semi-estructurada, técnica cualitativa que "se basa en una guía de asuntos o preguntas y el entrevistador tiene la libertad de introducir preguntas adicionales para precisar conceptos u obtener mayor información sobre los temas deseados" (Hernández et al., 2010, p. 410). A continuación, se presenta de forma esquemática las áreas exploradas en las entrevistas, llevadas a cabo con las juezas y psicólogas (se detallan en el apartado de Resultados).

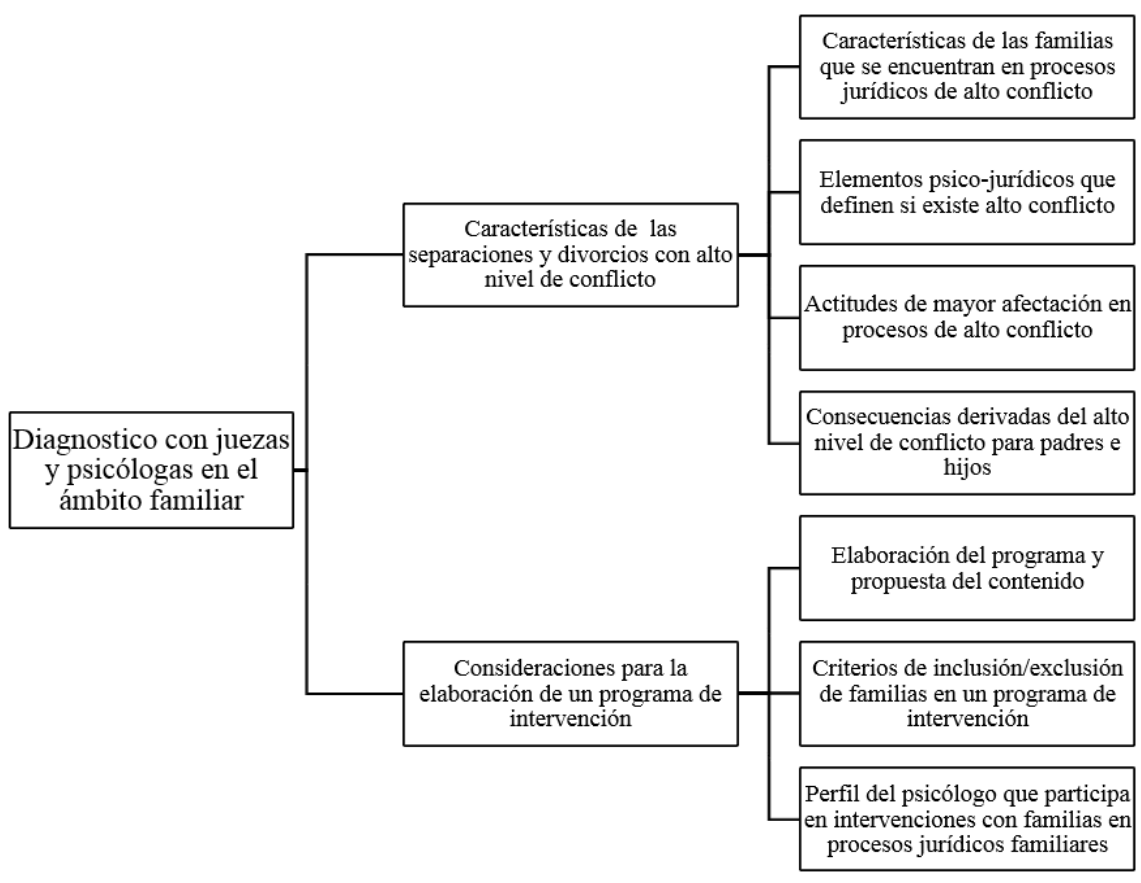

Figura 1. Áreas analizadas en la fase diagnóstica. 
Posterior a la aplicación de las entrevistas, se sintetizó la información en categorías específicas y se llevó a cabo un análisis para determinar los posibles objetivos, temas y beneficiarios del programa. Los resultados fueron obtenidos a través de los siguientes pasos propuestos para el análisis de datos cualitativos (Álvarez-Gayou, 2005; Miles \& Huberman, 1994; Rubin \& Rubin, 1995, citados en Fernández, 2006):

\section{Obtener la información}

2. Capturar, transcribir y ordenar la información

\section{Codificar la información \\ 4. Integrar la información}

\section{Resultados}

A continuación, se presentan los resultados obtenidos en las entrevistas individuales, las cuales fueron categorizadas a través de un análisis de los aspectos más relevantes y la frecuencia con la que éstos fueron mencionados por cada participante en las diferentes áreas exploradas.

\section{Tabla 1}

Características de las familias que se encuentran en procesos jurídicos familiares de alto conflicto

\begin{tabular}{ll}
\hline Categoría & Observaciones de los profesionales \\
\hline Características & Los padres presentan un pensamiento polarizado sobre los distintos aspectos del \\
divorcio & Se presenta mayor dificultad cuando existe nueva pareja o infidelidad \\
& Existe una ignorancia de derechos de los menores de edad y, por lo tanto, una \\
& afectación directa hacia ellos, al ser expuestos o utilizados dentro del conflicto \\
& Falta de acuerdos sobre la situación jurídica de los hijos (alimentos, custodia y \\
& convivencia) \\
& Las familias pueden presentar falta de estabilidad económica o, en su caso, \\
& acrecentar el conflicto cuando hay una lucha por el aspecto económico \\
\hline Problemáticas & Alienación parental \\
específicas que & Lucha de poderes \\
sugieren alto & Cuestiones emocionales sin resolver \\
nivel de conflicto & Falta de control de emociones \\
& Falta de capacidad para tomar acuerdos respecto a los hijos \\
& Resistencia y falta de afrontamiento positivo de la separación \\
& Poca o nulo asertividad para establecer comunicación entre las partes \\
& Dificultad para separar el proceso jurídico y los conflictos emocionales e \\
& interpersonales \\
& Baja autoestima \\
& Falta de acompañamiento adecuado a nivel legal, lo que detona mayor conflicto \\
\hline
\end{tabular}




\section{Tabla 2}

\section{Elementos psico-jurídicos que definen el alto nivel de conflicto}

\begin{tabular}{ll}
\hline Categoría & Observaciones de los profesionales \\
\hline Elementos & Junta de avenio: niveles de acuerdos y veracidad y falta para ponerse de acuerdo en \\
y pruebas & aspectos importantes \\
consideradas & Opinión del menor de edad ante el juez \\
para determinar & Periciales psicológicas \\
el alto nivel de & Periciales de trabajo social \\
conflicto & Alto nivel de litigio/múltiples denuncias \\
\hline Elementos & Existen conductas enmarcadas en la violencia familiar \\
psico-jurídicos & Existen conductas de alienación parental \\
que determinan & Se han suscitado hechos de retención o sustracción de menores de edad \\
el alto nivel de & Cuando se dificulta definir la situación jurídica del menor de edad \\
conflicto & Cuando existe afectación física, psicológica y social de los hijos menores de edad \\
& Se observa hostilidad entre las partes, descalificación o menosprecio \\
& La comunicación interparental es nula \\
& El tiempo en el que se ha mantenido el conflicto jurídico ha sido extenso \\
& Acusación de abuso sexual hacia los hijos \\
& Rechazo hacia algunos de los padres \\
& Interferencia en la convivencia con la madre o padre no custodio \\
& Pelea por dinero en familias con alto nivel económico \\
& Han intervenido demasiados profesionales en el desarrollo del proceso jurídico \\
\hline
\end{tabular}

\section{Tabla 3}

\section{Actitudes de mayor afectación en procesos de alto conflicto}

\begin{tabular}{ll}
\hline Categoría & Observaciones de los profesionales \\
\hline Actitudes & Violencia entre las partes y hacia los hijos \\
y patrones & Alguna de las partes no ha superado la ruptura \\
comportamentales & Cuando los padres no tienen apertura para tomar acuerdos en relación a los hijos \\
de mayor & Cuando no hay cumplimiento de las obligaciones hacia los hijos \\
afectación & Falta de empatía con las necesidades de los hijos \\
& Cuando los padres fomentan actitudes de rechazo y conductas de alienación parental \\
& Cuando existe falta de claridad en el proceso y mal acompañamiento y actuación de los \\
& profesionales involucrados
\end{tabular}




\section{Tabla 4}

Consecuencias que sufren las familias en procesos jurídicos con alto nivel de conflicto

\begin{tabular}{ll}
\hline Categoría & Observaciones de los profesionales \\
\hline Consecuencias & Conflicto de lealtades por parte de los hijos \\
del alto nivel & Hijos inmersos en el conflicto inter-parental \\
de conflicto & Niños influenciados (mentiras, enfermedades, trato y manipulación) \\
& Denuncias falsas de abuso sexual y violencia \\
& Rompimiento de la relación con el padre no custodio \\
& Menores de edad que dejan la escuela por falta de recursos económicos \\
& Descuido de las necesidades de los hijos \\
& Afectación psicológica y emocional tanto en los padres como en los hijos \\
& Desgaste emocional y físico \\
& Desgaste económico \\
& Alienación parental \\
& Violencia
\end{tabular}

\section{Tabla 5}

Consideraciones para el diseño e implementación de un programa de intervención para familias en procesos de alto conflicto

\begin{tabular}{|c|c|}
\hline Categoría & Observaciones de los profesionales \\
\hline $\begin{array}{l}\text { Consideraciones } \\
\text { para las fases de } \\
\text { intervención }\end{array}$ & $\begin{array}{l}\text { Llevar a cabo una intervención individual } \\
\text { Considerar la mediación (si es necesario) } \\
\text { Capacitación a los juzgadores para determinar criterios y objetivos de } \\
\text { intervención } \\
\text { Aplicar un diagnóstico que contemple a los participantes del conflicto (usuarios, } \\
\text { juzgadores, profesionales) } \\
\text { Contemplar el momento judicial en el que se encuentra } \\
\text { Dividir la intervención en etapas a partir de los objetivos y necesidades } \\
\text { Llevar a cabo una fase de seguimiento } \\
\text { Considerar la intervención de otro perfil profesional (trabajo social) }\end{array}$ \\
\hline $\begin{array}{l}\text { Contenido } \\
\text { (temáticas y áreas } \\
\text { de intervención } \\
\text { propuestas) }\end{array}$ & $\begin{array}{l}\text { Generales } \\
\text { Comunicación y asertividad } \\
\text { Regulación de emociones } \\
\text { Duelo } \\
\text { Rol parental } \\
\text { Necesidades y derechos de los hijos } \\
\text { Con los padres } \\
\text { Sobre el conflicto individual y aceptación de la separación } \\
\text { Conflicto interparental } \\
\text { Toma de acuerdos a nivel interparental } \\
\text { Comunicación entre padres e hijos } \\
\text { Convivencia entre padre no custodio e hijos } \\
\text { Estrategias de afrontamiento } \\
\text { Con los hijos (menores de edad) } \\
\text { Derechos y obligaciones de los niños } \\
\text { Conflicto parento-filial y afectaciones psicológicas y emocionales } \\
\text { Convivencia parento-filial } \\
\text { Manejo emocional ante el conflicto } \\
\text { Estrategias de enfrentamiento }\end{array}$ \\
\hline
\end{tabular}




\section{Tabla 6}

Criterios de inclusión/exclusión de familias a considerar en un programa de intervención

\begin{tabular}{ll}
\hline Categoría & Observaciones de los profesionales \\
\hline Criterios de & Existe dificultad de asumir rol paterno y se requiere orientación para ejercer el rol \\
inclusión & parental \\
& Se han lesionado los derechos de los hijos menores de edad \\
& El conflicto impide el desarrollo de las relaciones paterno-filiales \\
& No se han podido establecer acuerdos jurídicos sobre alimentos, convivencia y \\
& custodia \\
& Se dificulta reestablecer la relación parento-filial \\
& Se alega que hay maltrato infantil o violencia \\
& Existen comportamientos relacionados con la alienación parental \\
& Se observa la necesidad de contención emocional \\
& Cuando hay un nivel elevado de afectación a los menores de edad \\
\hline Criterios de & Cuando existe algún trastorno, alguna depresión, es necesario mandar a un programa \\
exclusión & alterno, depende del nivel de la patología \\
\hline
\end{tabular}

Tabla 7

Perfil del psicólogo que participa en intervenciones con familias en procesos jurídicos familiares

\begin{tabular}{ll}
\hline Categoría & Observaciones de los profesionales \\
\hline Perfil general & Conocimiento jurídico y en Derecho de familia \\
& Conocimientos de las problemáticas en contextos jurídicos familiares \\
& Conocimiento en intervención familiar y con menores de edad \\
& Especializado en psicología jurídica, evaluación psicológica en áreas jurídica y \\
& forense \\
& Conocimiento clínico \\
& Consideración de los lineamientos éticos (y legales) \\
\hline
\end{tabular}


A partir de este análisis, se vislumbra una serie de problemáticas o áreas a intervenir consideradas de mayor relevancia, desde la visión de los profesionales:

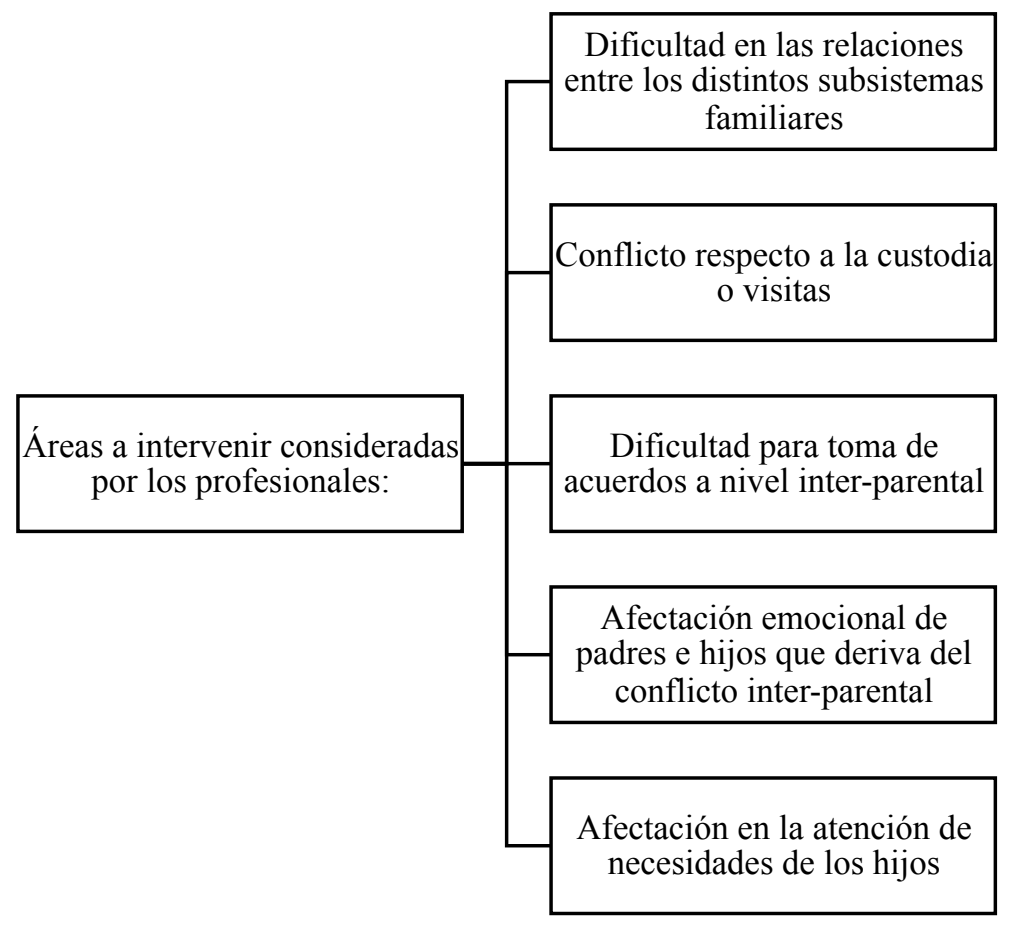

Figura 2. Problemáticas y áreas a intervenir desde la visión de los profesionales.

Los resultados sugieren la necesidad de intervenir desde el ámbito de la psicología jurídica con aquellas familias que se encuentren en divorcios conflictivos y que, por tanto, ponen en riesgo el desarrollo físico, psicológico, emocional y social de cada uno de sus miembros, principalmente el de las niñas y los niños. Las participantes del diagnóstico sugieren que es de gran importancia contar con estos programas y con la participación del psicólogo -desde la intervención-, para coadyuvar en la solución de los conflictos jurídico-familiares. De esta manera, se beneficia el cumplimiento de los derechos de cada miembro de la familia y se brindan herramientas para un mejor afrontamiento y bienestar individual. 


\section{Discusión}

El diagnóstico permitió describir las características de las familias que se encuentran en procesos jurídicos conflictivos. Lo cual es determinante al plantear la metodología de intervención, incluyendo fases, participantes, temas, técnicas y delimitar las necesidades de intervención.

En este sentido, la perspectiva de los profesionales en el ámbito familiar es congruente con lo señalado por diversos autores, por ejemplo, las entrevistadas consideran que los conflictos en relación con la custodia y el establecimiento del régimen de visitas son de mayor frecuencia en este tipo de conflictos. Así lo señalan Bonasa y colaboradores (2010), así como Bolaños (2002 como se cita en Tejedor, 2007), quienes consideran que en este tipo de separaciones suelen ser constantes la distorsión o manipulación de la imagen del otro progenitor, las interferencias para evitar que el otro cumpla el régimen de visitas y el imposibilitar la convivencia de forma contundente, a través de campañas de denuncias falsas, de la cronificación innecesaria del proceso judicial o cualquier otro tipo de comportamientos graves, además de los conflictos de lealtades y de invalidación del progenitor no custodio.

Sobre las relaciones inter-parentales, se encontró que la afectación se puede incrementar de forma significativa cuando existen problemáticas y conductas paternas, caracterizadas por la desatención de los hijos(as), ya que es difícil separar el proceso jurídico y los conflictos emocionales e interpersonales; son patentes, entonces, las dificultades para comunicarse y para concentrarse en las necesidades de los vástagos, así como dificultades para ubicar y proteger a los otros de su propia angustia emocional, ira o de las disputas constantes entre las partes. En tal contexto, se expresa una marcada falta de cooperación en las funciones parentales de cuidado, educación y bienestar de los hijos, como lo señala De la Torre (2006).

Asimismo, las juezas y psicólogas enfatizan las numerosas ocasiones donde se presentan situaciones de violencia, maltrato y agresión, lo que eleva el nivel de conflicto y daña directamente a los descendientes, como también lo comprueban Johnson y Roseby (1997), Cusó, (2005), Patró y Limiñana (2005) y Fariña, Arce, Seijo y Novo (2013), quienes puntualizan los efectos de la violencia, el maltrato infantil y las situaciones de riesgo en los hijos.

Entre otras de las afectaciones reseñadas por las juezas y las psicólogas, se encuentran la fuerte carga emocional, la mala adaptación a la ruptura y el impacto negativo en el bienestar psicológico de padres e hijos(as), estos aspectos, según Bolaños (1998), definen a su vez una separación como una situación de alto nivel de conflicto.

De acuerdo con lo expresado por las juezas y psicólogas, entre las problemáticas y consecuencias más relevantes para las familias en casos de divorcios conflictivos, se encuentran la dificultad en las relaciones entre los distintos 
subsistemas familiares, el conflicto respecto a la custodia o visitas, la falta de habilidades parentales y de resolución de problemas que garanticen los derechos de los miembros de la familia y el bienestar psicológico de cada uno, así como la incapacidad para tomar acuerdos entre los padres para el cuidado de los hijos.

En cuanto a los hijos, los principales problemas son la afectación emocional, que deriva del conflicto entre los padres; el deterioro en las relaciones parento-filiales por la falta de convivencia y por problemáticas específicas, como las interferencias parentales; $\mathrm{y}$, finalmente, la falta de atención y satisfacción de sus necesidades para su óptimo desarrollo.

Por otra parte, se observa que, tanto en padres como en hijos, existe la necesidad de adquirir pautas de afrontamiento y de convivencia, cuyo fin sea minimizar los conflictos familiares y generar un estado de bienestar tanto individual como familiar.

En consideración de estos resultados y atendiendo a las necesidades detectadas, se consideró importante plantear un programa que contemple la intervención con grupos de padres custodios y no custodios, e hijos menores de edad, de forma separada, con el fin de brindar una atención acorde a las problemáticas y necesidades que se presentan en cada grupo. Si bien las áreas de intervención son similares, las características por desarrollo y el tipo de afectación en cada grupo es diferente.

Por último, al concluir este diagnóstico, se estableció la necesidad de contar con un segundo acercamiento que evidencie las problemáticas surgidas en cada familia a partir de la intervención, esto con el propósito de brindar una atención acorde con las necesidades de las personas que serán seleccionadas.

Los resultados obtenidos respaldan la necesidad y la relevancia de aplicar programas especializados en el ámbito familiar, que tengan un impacto directo en el bienestar psicológico $\mathrm{y}$ emocional de las personas inmersas en el sistema de justicia y en la minimización de las afectaciones negativas provocadas por el nivel de conflicto, es decir, que aporten elementos para favorecer los procesos legales con un carácter más terapéutico, tal como señalan diversos autores (Tejedor, 2012, Fariña et al., 2002; Cantón, \& Justicia, 2007).

A modo de sugerencias y conclusiones, se hace énfasis en la importancia de tomar en cuenta la autonomía y derecho de una persona para decidir y aceptar su inclusión en un programa de tratamiento, sea este grupal o individual, lo cual entra en contraste con los ordenamientos judiciales de terapia que, muchas veces bajo la oficiosidad, obligan a las partes al cumplimiento de las ordenanzas.

Es importante que las autoridades judiciales puedan ejercer estos ordenamientos cuando son en beneficio de los niños afectados o que se encuentran en riesgo por las actitudes y el alto conflicto entre sus padres, sin embargo, cuando una persona no está dispuesta a aceptar este ordenamiento y no alcanza a comprender las implicaciones de este tipo de intervenciones, 
resulta contraproducente e incompatible con los objetivos que se pretenden alcanzar.

Para concluir, es relevante resaltar el aspecto ético, fundamental al momento de plantear este tipo de intervenciones especializadas, puesto que exige un apego estricto a la ética de la profesión y la legalidad en la que se enmarca. Además, es altamente relevante la protección y el respeto a la dignidad de cada una de las personas participantes, tomando en cuenta su sentir, su realidad y sus necesidades, para poder finalmente incidir en el acceso a una justicia más terapéutica y más humana.

\section{Referencias}

Ackerman, J. (1995). Clinician's Guide to Child Custody Evaluations. Nueva York: John Wiley and Sons.

Aguilar, J. (2007). Interferencia de las Relaciones Paterno Filiales, el Síndrome de Alienación Parental y las Nuevas Formas de Violencia contra la Infancia. Psicología Educativa, 13(2), 101-116.

Arce, R., \& Fariña, F. (2007). Un programa de intervención con menores en riesgo por desestructuración familiar. En F. J. Rodríguez \& C. Becedoniz (Coords.), El menor Infractor. Posicionamientos y realidades (pp. 264-279). España: Principado de Asturias/ Colección Ley y Ciencias Sociales.

Arch, M. (2010). Divorcio conflictivo y consecuencias en los hijos: implicaciones para las recomendaciones de guarda $\mathrm{y}$ custodia. Papeles del Psicólogo, 31(2), 183-
190. Recuperado de http://www.redalyc.org/ articulo.oa? $\mathrm{id}=77813509004$

Bolaños, J. I. (2000). Estudio descriptivo del síndrome de alienación parental en procesos de separación y divorcio. Diseño y aplicación de un programa piloto de mediación familiar (Tesis doctoral, Facultad de Psicología, Universidad Autónoma de Barcelona). Recuperado de http:/www.tdx. cat/handle/10803/4733

Bolaños, J. I. (1998). Conflicto familiar y ruptura matrimonial: Aspectos psicolegales. En J. L. Marrero (Comp.), Psicología Jurídica de la familia (43-76). Madrid: Fundación Universidad Empresa/Retos jurídicos en las Ciencias Sociales.

Bonasa, M., López, J., Cartil, C., Punset, V., Espada, M., Cortés, R., \& Checa, M. (2010). Procesos disfuncionales en procedimientos de separación y divorcio. En F. Fariña, R. Arce, M. Novo \& D. Seijo (Coords.), Separación y Divorcio: interferencias parentales (pp. 103114). España: ASEMIP.

Cantón, J., Cortés, M. R., Justicia, M. D., \& Cantón, D. (2014). Violencia doméstica, divorcio y adaptación psicológica. Madrid: Ediciones Pirámide.

Cantón, J., \& Justicia, M. D. (2007). Programas para prevenir los problemas de adaptación de los hijos de divorciados. En J. Cantón, M. R. Cortés \& M. D. Justicia (eds.), Conflictos entre los padres, divorcio y desarrollo de los hijos (pp. 243-276). Madrid: Ediciones Pirámide. 
Cortés, M. R. (2010). Adaptación psicológica de los hijos al divorcio de los padres. En R. Arce, F. Fariña, M. Novo \& D. Seijo (Coords.), Separación y divorcio: interferencias parentales (pp. 57-76). España: ASEMIP.

Cusó, M. (1995). Infancia en riesgo e infancia maltratada. Comunicación, lenguaje $y$ educación, (27), 87-96.

De la Torre, J. (2005). Las relaciones entre padres e hijos después de las separaciones conflictivas. Apuntes de Psicología, 23(1), 101-112.

De la Torre, J. (2006). Los puntos de encuentro familiar: un enfoque actual de intervención en situaciones de ruptura familiar. Anuario de Psicología Jurídica, 16, 65-73.

Deutsch, R. M. (2008). Divorce in the 21st century: Multidisciplinary family interventions. Journal of Psychiatry and Law, (36), 41-67.

Fariña, F., \& Arce, R. (2006). El papel del psicólogo en casos de separación o divorcio. En J. C. Sierra, E. M. Jiménez \& G. BuelaCasal (Coords.), Psicología forense: Manual de técnicas y aplicaciones (pp. 246-271). Madrid: Biblioteca Nueva.

Fariña, F., Arce, R., Seijo, M., \& Novo, M. (2013). El hijo como víctima de los problemas de pareja: Un abordaje desde la justicia terapéutica. En S. P. Colín, E. GarcíaLópez \& L. A. Morales (Coords.), Ecos de la violencia, voces de la reconstrucción ( $\mathrm{pp}$. 49-72). México: Servicio de Publicaciones de la Universidad de Morelia.
Fariña, F., Seijo, D., Arce, R., \& Novo, M. (2002). Psicología Jurídica de la Familia: Intervención en casos de Separación y Divorcio. Barcelona: Cedecs.

Fernández, E., \& Godoy, C. (2005). El niño ante el divorcio. Madrid: Ediciones Pirámide.

Fernández, L. (2006). ¿Cómo analizar datos cualitativos? Butlletí La Recerca, (7). Recuperado de http://www.ub.edu/ice/ recerca/pdf/ficha7-cast.pdf

Frías-Armenta, M. (Diciembre 2014). Justicia Terapéutica en México. En D. B. Wexler, F. Fariña, L. A. Morales \& S. P. Colín. (Comps.). Justicia terapéutica: Experiencias y aplicaciones (pp. 29-38). Puebla: Inacipe.

Gil, A. (2011). Acciones del sistema nacional DIF para prevenir la alienación parental: crianza humanizada y parentalidad bientratante. En Comisión Nacional de los Derechos Humanos, Alienación Parental (pp. 269-286). México: Comisión Nacional de los Derechos Humanos.

Hernández, R., Fernández, C. \& Baptista, P. (2010). Metodología de la investigación. México: McGraw-Hill.

Johnston, J., \& Roseby, V. (1997). In the name of the child: A developmental approach to understanding and helping children of conflicted and violent divorce. Nueva York: The Free Press.

Kirby, J. J. (1998). Court-related parenting education divorce interventions. Human Development and Family Life Bulletin. A Review of Research and Practice, 4(2), 1-3. 
Muñoz, A. (2009). Personas Divorciadas: Análisis de las Características del Proceso de Ruptura. Intervención Psicosocial, 18(1), 65-74.

Patró, R., \& Limiñana, R. M. (junio, 2005). Víctimas de violencia familiar: consecuencias psicológicas en hijos de mujeres maltratadas. Anales de Psicología, 21(1), 11-17.

Tejedor, M. A. (2007). Intervención ante el síndrome de Alienación Parental. Anuario de Psicología Jurídica, 17, 79-89.

Tejedor, M. A. (2010). Intervención en casos de alienación parental. En F. Fariña, R. Arce, M. Novo \& Seijo, D. (Eds.), Separación y Divorcio: interferencias parentales (pp. 133147). España: Asemip.

Tejedor, M. A. (2012). El interés de los menores en los procesos contenciosos de separación o divorcio. Anuario de Psicología Jurídica, 22, 67-75. Recuperado de http://www.redalyc. org/articulo.oa?id=315024813007

Recibido el 20 de noviembre de 2017

Revisado el 24 de noviembre de 2017

Aceptado el 27 de noviembre de 2017 